\title{
Angstrom-scale Magnetic Measurements of a Metallic Antiferromagnet with 4D- STEM
}

Jeffrey Huang ${ }^{1}$, KAYLA NGUYEN ${ }^{2}$, Manohar Karigerasi ${ }^{1}$, Kisung Kang ${ }^{1}$, André Schleife ${ }^{1}$, Daniel Shoemaker ${ }^{1}$, David Cahill ${ }^{1}$, Jian-Min $\mathrm{Zuo}^{3}$ and Pinshane Huang ${ }^{1}$

${ }^{1}$ University of Illinois at Urbana-Champaign, United States, ${ }^{2}$ University of Illinois at Urbana-Champaign, URBANA, Illinois, United States, ${ }^{3}$ University of Illinois at Urbana-Champaign, Urbana, Illinois, United States

Antiferromagnets have recently attracted significant interest for their potential use in spintronic devices. Due to their switching frequencies in the terahertz range, antiferromagnets have the potential to produce fast, dense computer memories[1]. Existing magnetic imaging techniques such as Lorentz TEM[2], electron holography[3,4], and differential phase contrast scanning transmission electron microscopy (DPC-STEM)[5,6] have been used effectively to image magnetic domains and topological structures of magnetism such as skyrmions[7] with spatial resolution of a couple of nanometers[7,8]. However, higher spatial resolution is needed to study the spin arrangements of antiferromagnets near domain boundaries, defects, and interfaces.

In this work, we demonstrate experimental 4D-STEM methods, developed with multislice simulations, to image the local magnetic moments in $\mathrm{Fe}_{2} \mathrm{As}$, a metallic antiferromagnet. $\mathrm{Fe}_{2} \mathrm{As}$ is an ideal sample because the $\mathrm{Fe}$ atoms are organized in $6 \AA$-wide blocks in which the spins are locally aligned. The magnetic moments of the Fe atoms are parallel within the same block and reverse direction in adjacent blocks, forming a pattern that repeats every $11.96 \AA$ (Fig. 1) [9].

First, we utilize quantitative electron scattering simulations to understand the effects of magnetism on convergent beam electron diffraction (CBED) patterns and determine ideal experimental parameters to detect magnetism with an atomic-scale probe. We use conventional multislice simulations[10] with a modification: we include a magnetic phase shift in the transmission function for each slice, using magnetization densities calculated for $\mathrm{Fe}_{2} \mathrm{As}$ from density-functional theory. From our simulations, we found that the magnetic signal was maximized when the real-space probe matched the size of the $6 \AA$ wide blocks in which the spins are aligned, or equivalently when the Bragg disks overlap slightly. Our simulations also show that the magnetic structure of $\mathrm{Fe}_{2} \mathrm{As}$ causes redistribution of intensity within the bright field disk in addition to small (microradian) shifts of the diffraction pattern.

Guided by our multislice simulations, we optimized methods to extract and isolate a magnetic signal. We defined a circular mask (Fig. 2b) in the center of the bright field disk and used the masked area to produce center of mass (COM) images from experimental CBED patterns. As seen in Figs. 2c-2e, the component of those COM images along the [001] direction shows spatial variation at half the spatial frequency of the (001) lattice fringes, consistent with the magnetic unit cell of $\mathrm{Fe}_{2} \mathrm{As}$. This data indicates that our methods can directly measure magnetism in antiferromagnetic $\mathrm{Fe}_{2} \mathrm{As}$ with $6 \AA$ A resolution. Together, our simulation and experimental methods illustrate new methods for electron microscopy measurements of antiferromagnetic materials down to few-angstrom resolution, and we expect that these techniques should be useful in the development of spintronic devices. [11] 
a)

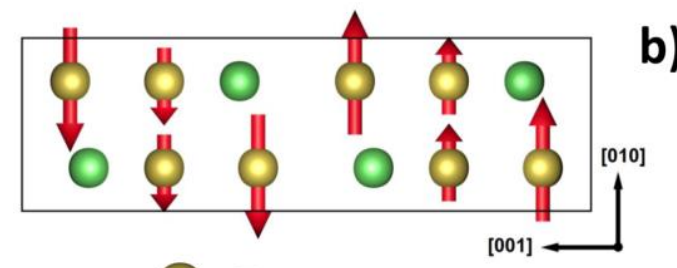

$\mathrm{Fe}$

As

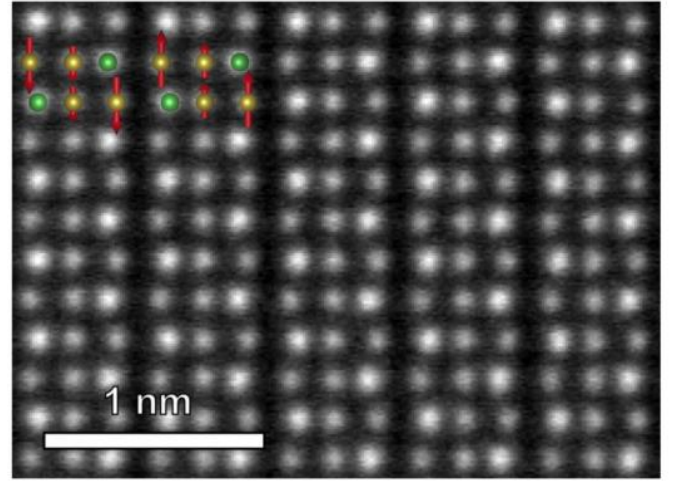

Figure 1. Structure of $\mathrm{Fe}_{2} \mathrm{As}$. a) Magnetic unit cell of $\mathrm{Fe}_{2} \mathrm{As}$, showing Fe atoms (gold) and As atoms (green). Magnetic moments of Fe atoms are indicated by arrows. b) ADF-STEM image of $\mathrm{Fe}_{2} \mathrm{As}$.

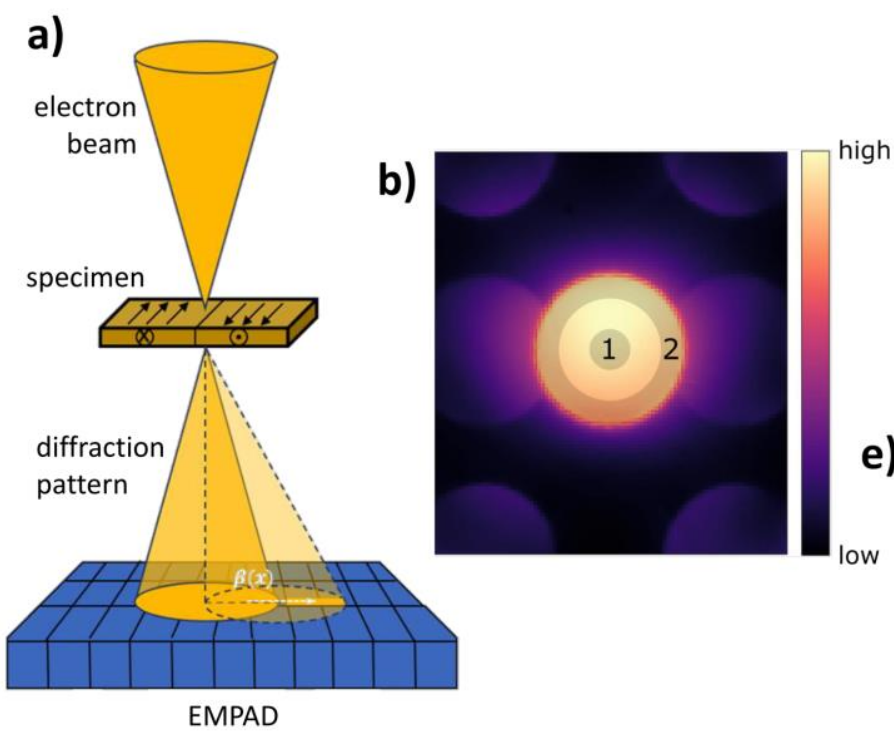

c)

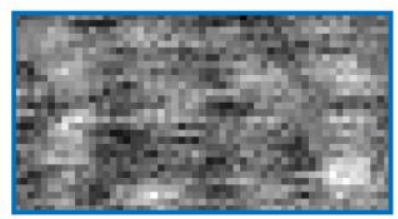

d)

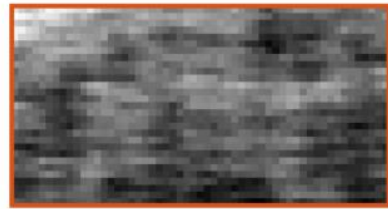

e)

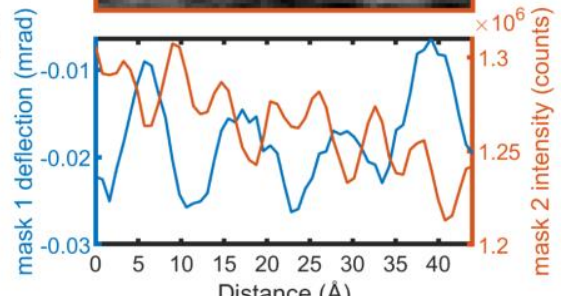

Figure 2. a) Schematic showing how sample magnetism causes small deflections to the electron beam. b) Experimental PACBED image of $\mathrm{Fe}_{2} \mathrm{As}$. Dark transparent regions define a circular mask (1) and an annular mask (2). c) and d) Images of same region of $\mathrm{Fe}_{2} \mathrm{As}$ sample, with scale indicated in e). c) Center of mass image (component in the [001] direction) using mask 1, showing spatial variation consistent with magnetic structure of $\mathrm{Fe}_{2} \mathrm{As}$. d) Image summed over mask 2, showing lattice fringes. e) Line profiles of c) and d).

\section{References}

[1] T. Jungwirth, J. Sinova, A. Manchon, X. Marti, J. Wunderlich, and C. Felser, Nat. Phys., vol. 14, no. 3, pp. 200-203, 2018.

[2] A. K. Petford-Long and M. De Graef, in Characterization of Materials, Hoboken, NJ, USA: John Wiley \& Sons, Inc., 2012, pp. 347-369.

[3] M. Mankos, M. R. Scheinfein, and J. M. Cowley, in Handbook of Nanoscopy, vol. 1, Weinheim, Germany: Wiley-VCH Verlag GmbH \& Co. KGaA, 1996, pp. 323-426.

[4] T. Tanigaki et al., Microsc. Microanal., vol. 25, no. S2, pp. 54-55, 2019. 
[5] J. N. Chapman, P. E. Batson, E. M. Waddell, and R. P. Ferrier, Ultramicroscopy, vol. 3, no. C, pp. 203-214, 1978.

[6] K. Müller et al., Nat. Commun., vol. 5, 2014.

[7] D. McGrouther et al., New J. Phys., vol. 18, no. 9, 2016.

[8] K. X. Nguyen et al., arXiv: 2001.06900, Jan. 2020.

[9] H. Katsuraki and N. Achiwa, J. Phys. Soc. Japan, vol. 21, no. 11, pp. 2238-2243, Nov. 1966.

[10] J. M. Cowley and A. F. Moodie, Acta Crystallogr., vol. 10, no. 10, pp. 609-619, 1957.

[11] This work was supported by AFOSR grant number AF FA9550-20-1-0302 and NSF-MRSEC award number DMR-1720633. Experiments were carried out in the Cornell Center for Materials Research Shared Facilities (supported through the NSF MRSEC program (DMR-1719875)) and in the Materials Research Laboratory Central Research Facilities at the University of Illinois. 